\title{
Caracterización de los controles ambientales de una empresa de producción de plástico del municipio de Caçador-SC, Brasil
}

\author{
Characterization of the environmental controls of a plastic production company of the municipality of Caçador-SC, \\ Brazil
}

\author{
Roger Francisco Ferreira de Campos ${ }^{1}$, Tiago Borga² e Santiago Sánchez García ${ }^{3}$ \\ ${ }^{1}$ Maestría, Ingeniero Ambiental y Sanitario, Universidade do Estado de Santa Catarina, Lages, Santa Catarina, Brasil \\ roger@uniarp.edu.br
}

${ }^{2}$ Maestría, Ingeniería Ambiental, Universidade Alto Vale do Rio do Peixe, Caçador, Santa Catarina, Brasil tiagoborga@gmail.com

${ }^{3}$ Graduando, Ingeniería Civil y territorial, ETSI Caminos, Canales y Puertos, Ciudad Real, España santi21sanchez@hotmail.com

\begin{abstract}
Resumen
Muchos procesos productivos poseen un alto nivel de emisión de aspectos e impactos ambientales. Para buscar la minimización de esos atenuantes ambientales se desarrolló la licencia ambiental que emplea los controles ambientales de las empresas. Así, el presente trabajo tiene como principal objetivo analizar la caracterización de los controles ambientales de una empresa de producción de productos derivados del plástico en el municipio de Caçador/SC. Para el desarrollo del estudio se analizaron los controles establecidos en la Licencia Ambiental de Operación - LAO para realizar la caracterización in situ y documentación de los controles ambientales. A través del estudio es posible describir que la empresa está en conformidad con las cuestiones ambientales, ya que la empresa busca minimizar al máximo los impactos generados. Así, se puede concluir que la caracterización de los controles ambientales es un método que ayuda en la minimización de los impactos ambientales, donde el mismo presenta los puntos positivos y negativos de la interacción del proceso productivo con el medio ambiente.
\end{abstract}

Palabra clave: Controles ambientales. Impacto medioambiental. Gestión ambiental de empresas

\begin{abstract}
Many production processes have a high level of emission of environmental aspects and impacts, in order to seek the minimization of these environmental mitigators, the environmental license that employs the environmental controls of the companies was developed. The main objective of this paper is to analyze the characterization of the environmental controls of a company producing plastic products in the municipality of Caçador/SC. For the development of the study, the controls established in the Environmental Operating License (EOL) were analyzed to carry out in situ and documentary characterization of environmental controls. Through the study it is possible to describe that the company is in compliance with environmental issues, since the company seeks to minimize the impacts generated. Thus, it can be concluded that the characterization of environmental controls is a method that helps to minimize environmental impacts, where it presents the positive and negative points of the interaction of the productive process with the environment.
\end{abstract}

Keyword: Environmental controls. Environmental impact. Environmental management of companies 


\section{INTRODUCCIÓN}

La búsqueda para el desarrollo sostenible de las empresas y una interacción positiva con las cuestiones ambientales está atribuida a la percepción de prácticas ambientales como la implantación de procedimientos que proporcionen una gestión ambiental satisfactoria, viabilizando los procesos productivos ante los sistemas ambientales (SELBACH; NAIME, 2014).

Según Lazarotto et al., (2015) muchas empresas tienen interés en preservar la calidad del medio ambiente, sin embargo, este proceso no es muy perceptible en los procesos productivos, ya que muchas veces falla, por falta de supervisión y conocimiento sobre el proceso que produce impacto ambiental, acarreando problemas ambientales.

Entre los procesos productivos es posible analizar diferentes fuentes de contaminación, donde se pueden destacar los efluentes industriales (PEREIRA, 2004; SANTOS et al., 2010), agotamiento sanitario (SILVA; PEREIRA, 2014), emisión de gases (CAMPOS; COSTA, 2017), residuos sólidos no peligrosos (PAZ; MORAIS; HOLANDA, 2013), residuos sólidos peligrosos (SISINNO, 2003; BRAGA et al., 2005) y ruido (GIRARDI; SELLITTO, 2011).

D’Agostin et al., (2017) describen que la degradación ambiental parte de las actividades antrópicas, que en muchas situaciones, no puede ser evitada; sin embargo se deben buscar medios para minimizar o mitigar el proceso de degradación buscando siempre una interacción de forma sostenible en el medio proceso productivo del hombre. Según Anjos et al., (2017) el desarrollo de medios para la minimización de los impactos ambientales en área urbana se hace necesario, ya que la interacción del crecimiento de áreas urbanas para el desarrollo del hombre está afectando a la calidad ambiental. Para Leandro y Neffa (2014) es necesario desarrollar tecnología y utilizar los medios para disminuir los impactos ambientales y, consecuentemente, minimizar los efectos de la crisis ambiental por la que estamos pasando, tanto en Brasil como en el resto del mundo.

La implantación de la Gestión Ambiental - GA proporciona un ambiente de mejor calidad, más limpio y seguro, proporcionando un mejor rendimiento debido a la calidad del ambiente de trabajo y, consecuentemente, reduciendo los impactos negativos como el desecho irregular de residuos, además de adecuar a la empresa con las leyes ambientales pertinentes a los procesos (LAZAROTTO et al., 2015). La cuestión de la responsabilidad socioambiental de una empresa puede ser vista como un método de diferencia en el mercado, pues una empresa de acuerdo con las cuestiones medioambientales es mejor vista por los consumidores que una empresa que no presenta esta cuestión (BORELLA; NAIME, 2012; MONARO et al., 2015), además de estar en conformidad con los órganos ambientales (BOGARIM et al., 2014) y presentar generación de recursos (CAMPOS; LIMA, 2014).

Para Halberstadt et al., (2015) la práctica de los controles ambientales de una empresa está relacionada a menudo con los cobros externos, no estando relacionadas con las cuestiones ambientales de la empresa, ya que, a día de hoy, conforme a las legislaciones establecidas y reguladas por los órganos de fiscalización ambiental están haciendo que las empresas sufran costos sobre los controles ambientales, resultando el control de los residuos como uno de los principales, aunque esta cuestión es incipiente en Brasil (COSTA; SOARES; PESSOAS, 2015). Silva et al., (2002) añade que estas cuestiones no pueden ser analizadas como una obligación ante las legislaciones sino como un método que viabilice la producción, posibilitando la reducción de costos, aumento de productividad y desarrollo de la región determinada donde está insertado el sector de la empresa.

Las etapas de la reducción de la cantidad de contaminantes lanzados al medio ambiente están asociadas con las políticas ambientales dirigidas por las empresas debido a las medidas registradas tanto por una red de monitoreo ambiental como por un sistema de recolección de indicadores previamente existentes o definidos e implantados, esta cuestión se atribuye a la legislación pública y los sistemas de gestión ambiental, teniendo en cuenta que el proceso de gestión está íntimamente relacionado con el proceso de controles ambientales de las empresas (BRAGA et al., 2005).

De acuerdo con Bernardo y Camarottob (2012) la identificación de los controles ambientales es una práctica necesaria, dado que presentan un ejemplo de soluciones reales para minimizar y disminuir los impactos ambientales, pudiendo servir de referencia para otras empresas que busquen la adecuación ambiental y el desarrollo sostenible.

Por lo tanto, el presente trabajo tiene como objetivo desarrollar un estudio de caso, a través de un levantamiento de datos in situ de los controles ambientales de una empresa de reciclaje de plás- 
tico, con la perspectiva de caracterizar las medidas de minimización de impacto ambiental producido mediante el proceso productivo.

\section{MATERIALES Y MÉTODO}

\section{ZONA DE ESTUDIO}

La zona de estudio es una empresa de producción de materiales plásticos ubicada en el municipio de Caçador, Santa Catarina, conforme Figura 1. La empresa tiene como principal actividad la producción de bolsas, bobinas plásticas y otros, provenientes de polietileno y propileno. La empresa está sobre actividad (22.22-06-00) - Fabricación de embalajes de material plástico (CONSEMA, 2013), siendo una de las principales empresas del ramo en el estado de Santa Catarina.

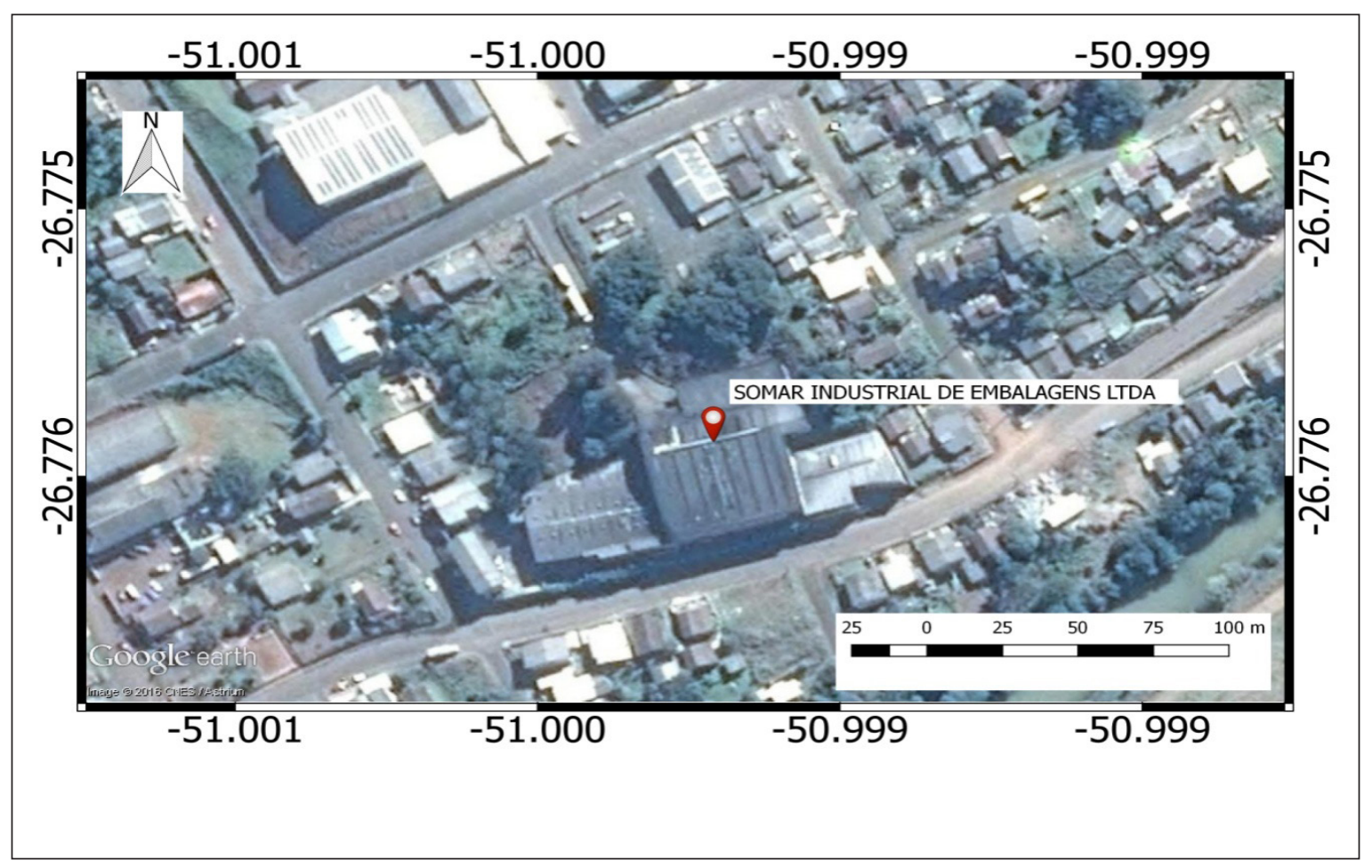

Figura 1 - Zona de estudio

Fuente: Autores (2017)

La empresa del estudio posee un terreno de $11.000 \mathrm{~m}^{2}$, una superficie construida de $4299,43 \mathrm{~m}^{2}$ y área libre de $5,000 \mathrm{~m}^{2}$. Posee tres turnos de trabajo de lunes a domingo. Presentando una producción media de 200 ton.mes-1 de embalajes plásticos flexibles, conforme Tabla 1. La empresa del estudio trabaja en tres turnos de lunes a domingo con diferentes horarios, a través de 50 empleados, la cual respeta la integridad de los empleados y está de acuerdo con las leyes laborales.

Tabla 1 - Cantidad de materia prima procesada por empresa de estudio

\begin{tabular}{c|c|c|c}
\hline \multicolumn{4}{|c|}{ CANTIDAD DE MATERIA PRIMA PROCESADA } \\
\hline Producto & Cantidad utilizada & Procedencia & Unidad de medida \\
\hline$>$ & 200 & Brasileña & ton.mes ${ }^{-1}$ \\
\hline
\end{tabular}

* Películas y embalajes plásticos flexibles. 


\section{Proceso PRODUCTIVO}

La primera etapa del proceso productivo está constituida por las Ventas. El representante comercial envía el Excel del pedido al sector de ventas donde se hará el análisis crítico y confirmación del pedido. En la segunda etapa se produce el POP (Pedido de Orden de Producción), donde se solicita una cantidad específica de materia prima, y se hace la programación del pedido en las máquinas en los sectores de extrusión, impresión, refine, laminación y corte soldadura.

En la tercera etapa ocurre el proceso de extrusión, donde el coordinador del sector recibe la Orden de Producción, preparan a la materia prima y abastecen el embudo de la extrusora. Preparan la máquina, inician la producción del artículo. Los operadores, encargados y monitores de la calidad inspeccionan el seguimiento durante el proceso. Realizan ajustes en caso de irregularidades, identifican el material y separan para los siguientes pasos. (Extrusión material en forma de bobinas) (Impresión, Laminación, Refile, Corte/Soldadura, Reembalaje, Expedición).

En la cuarta etapa, ocurre la impresión, donde si el material es impreso, donde ya está programado en qué máquina va a rodar $4,6 \mathrm{u} 8$ colores, siendo el coordinador del sector el que dirige las órdenes y entrega al encargado del turno, junto con los estándares todos conferidos. En ese momento se prepararán las tintas utilizadas en la producción del ítem, proveen cinta doble cara, doctorblade, cilindro, anilox y bobina de acierto. El operador hará su trabajo, realizará ajustes finales, tales como encajes, paso de fotocélula, texto y el color o el ajuste de la tonalidad conforme solicitud del cliente. Hecho esto el operador coloca la bobina del pedido en la máquina, toma una muestra para comprobación de la monitora de la calidad. Hecha la comprobación de todas las especificaciones del cliente junto a la muestra la monitora autoriza o no al operador a girar a la máquina. Una vez que esté girando, se toma una muestra de cada bobina impresa para monitoreo responsable para realizar el análisis final y archivar las mismas. Los técnicos identifican el material después del término del proceso y separan el material para los próximos pasos.

En la quinta etapa, ocurre la laminación, donde si el material es laminado, el operador y monitores de calidad reciben el patrón, ficha técnica y orden de producción. El operador prepara la máquina, ajusta la dosificación de adhesivo y el catalizador. Comprueba si la película tiene tratamiento y realizan pruebas de peso. Realizan inspecciones de seguimiento durante el proceso. En caso de irregularidades se realizan los ajustes. Después de esto, identifican las bobinas y las disponen para el tiempo de curación. Después de este tiempo, separan el material para los siguientes pasos.

En la sexta etapa ocurre el refile, donde si el material es refilado, reciben el patrón y orden de producción. Realizan los ajustes en la máquina, accionan, comprueban que todas las bobinas están con las medidas correctas, regulan la velocidad de la máquina para controlar los metros de película refilados, realizan inspecciones de acompañamiento durante el proceso. Después de terminar el proceso realizan el correcto almacenamiento, y se separan para los próximos pasos.

En la séptima etapa se produce el corte y la soldadura, donde el coordinador recibe la orden de operación estándar, conforme a los requisitos de la orden de producción, y verifican por qué maquina el material debe pasar: soldadura lateral, soldadura de fondo, soldadura lateral con vaina, soldadura superior/tubo, soldadura dorso o picadora. Se realizan inspecciones durante el proceso, se realizan pruebas de soldadura y resistencia en los embalajes. Embalan el material según la cantidad requerida por el cliente y separan para el siguiente paso.

En la octava etapa se produce la expedición, donde se almacena el material por rutas.

En la novena y última etapa ocurre la facturación, donde se reciben los datos del pedido y se aguarda la confirmación del término del mismo por el coordinador del sector. Después del término del proceso productivo, el coordinador del sector comunica la facturación sobre qué fecha la solicitud estará expuesta. Durante y después del término del pedido, toda la comunicación con el cliente sobre el mismo es realizada por la facturación. Una vez que el material esté aguardando el embarque para el cliente, la facturación emite la factura. El material será entregado por Somar o por un transportista tercero. Si el material es entregado por el transportista, la facturación solicita la recogida junto a la misma. 


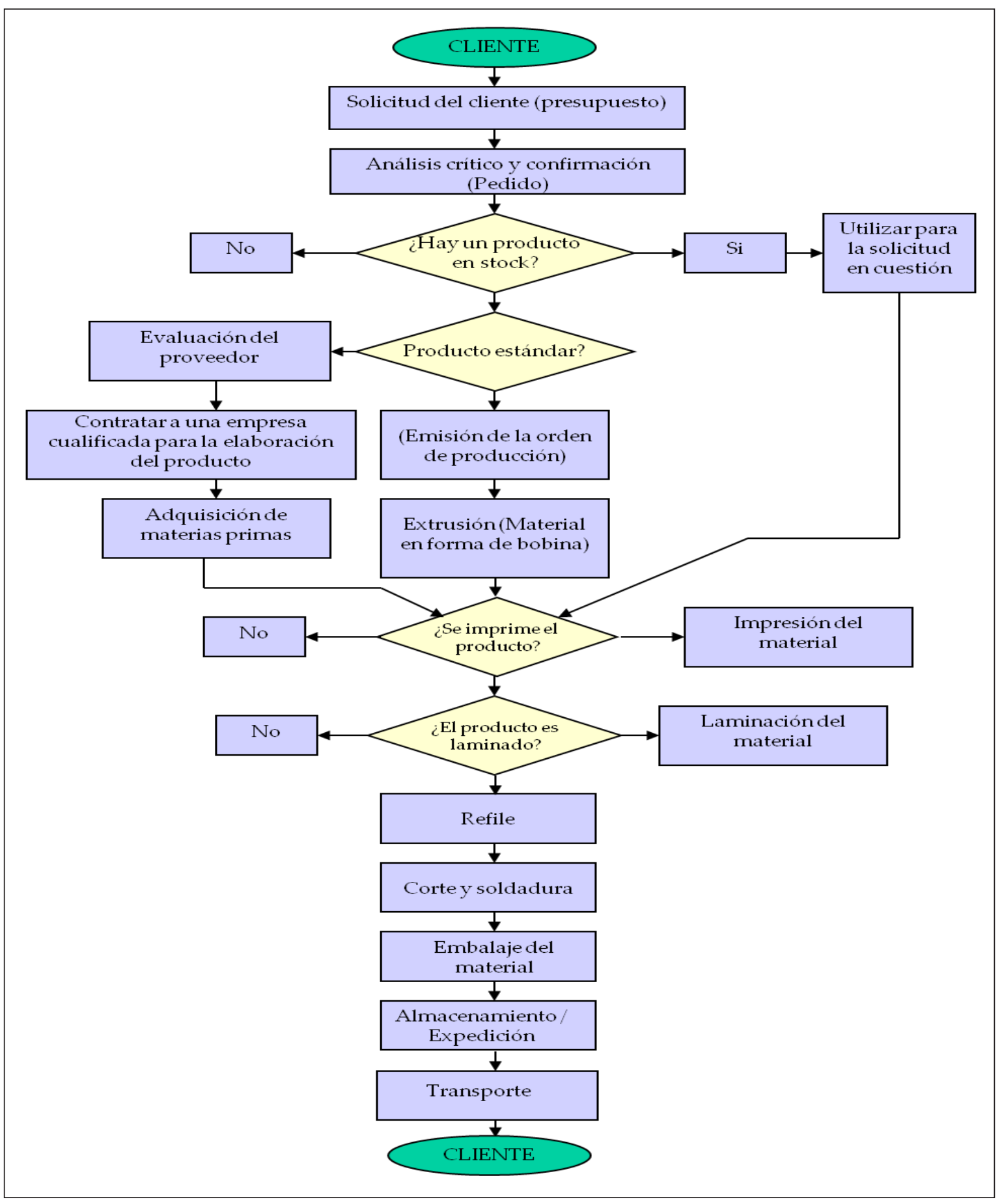

Figura 2 - Flujograma del proceso productivo;

\section{Proceso de ANÁLisis de los CONTROLES AMBientales}

Para que el objetivo fuera saneado fue realizado el análisis de los controles ambiental entre el año 2016 y 2017, para ese proceso se siguió lo establecido en la Licencia Ambiental de Operación - LAO de $n^{\circ}$ 6858/2011 de la empresa, a través del proceso de generación de residuos, tratamiento de residuos efluentes y agotamiento sanitario, reaprovechamiento el agua de lluvia y generación de solventes. Los análisis fueron seguidos de levantamiento in situ de los controles ambientales descrito en la LAO, con excepción de los residuos que fueron levantados conforme a las notas fiscales y el Manifiesto de Transporte de Residuos - MTR, así como a través del comparativo con las normas vigentes y legislaciones pertinentes al asunto abordado. 


\section{RESULTADOS Y DISCUSIÓN}

\section{EFLUENTES SANITARIO}

El efluente sanitario de la empresa es tratado por el sistema de fosa séptica de 4,580 litros (Diametro de 1,70 metros) y filtro anaerobio 4.480 litros (Diametro de 1,70 metros) estando de acuerdo con la NBR 7229 (ABNT, 1993) La limpieza es realizada según sea necesario por una empresa debidamente licenciada entrenada para recoger y tratar efluentes sanitarios, en el antento una limpieza se realiza anualmente según lo solicitado por la FATMA. De acuerdo con Whitman, Mihelcic y Mayer (2015) el sistema de tratamiento de aguas residuales de sanitario está asociado con el número de usuarios, el clima y la economía local. Por lo tanto, la empresa del estudio presenta conformidad ambiental sobre ese parámetro, ya que la misma está dimensionada conforme a las legislaciones pertinentes, pues presenta la aprobación del órgano de fiscalización sanitaria del municipio. Para Pimenta et al., (2002) es necesario invertir en el proceso de gestión y tratamiento de efluentes sanitarios, por ser una cuestión que permite el desarrollo sostenible. Aunque la fosa y el filtro no son eficientes en el tratamiento, se realiza la gestión de la misma, con limpieza y mantenimiento, buscando la mejor eficiencia de ese sistema.

\section{REAPROVECHAMIENTO DE AGUA}

La empresa tiene los procesos de reaprovechamiento de agua, iniciando el primer proceso constituido por el aprovechamiento del agua de lluvia para el proceso industrial, hidrantes y vasos sanitarios - con la capacidad de 25.000 litros que atiende a la empresa en tiempos de sequía, no con el fin volumen necesario, según figura 1 . El segundo está constituido por un proceso de enfriamiento del efluente que se forma en las instrusoras, De acuerdo con la Figura 2.

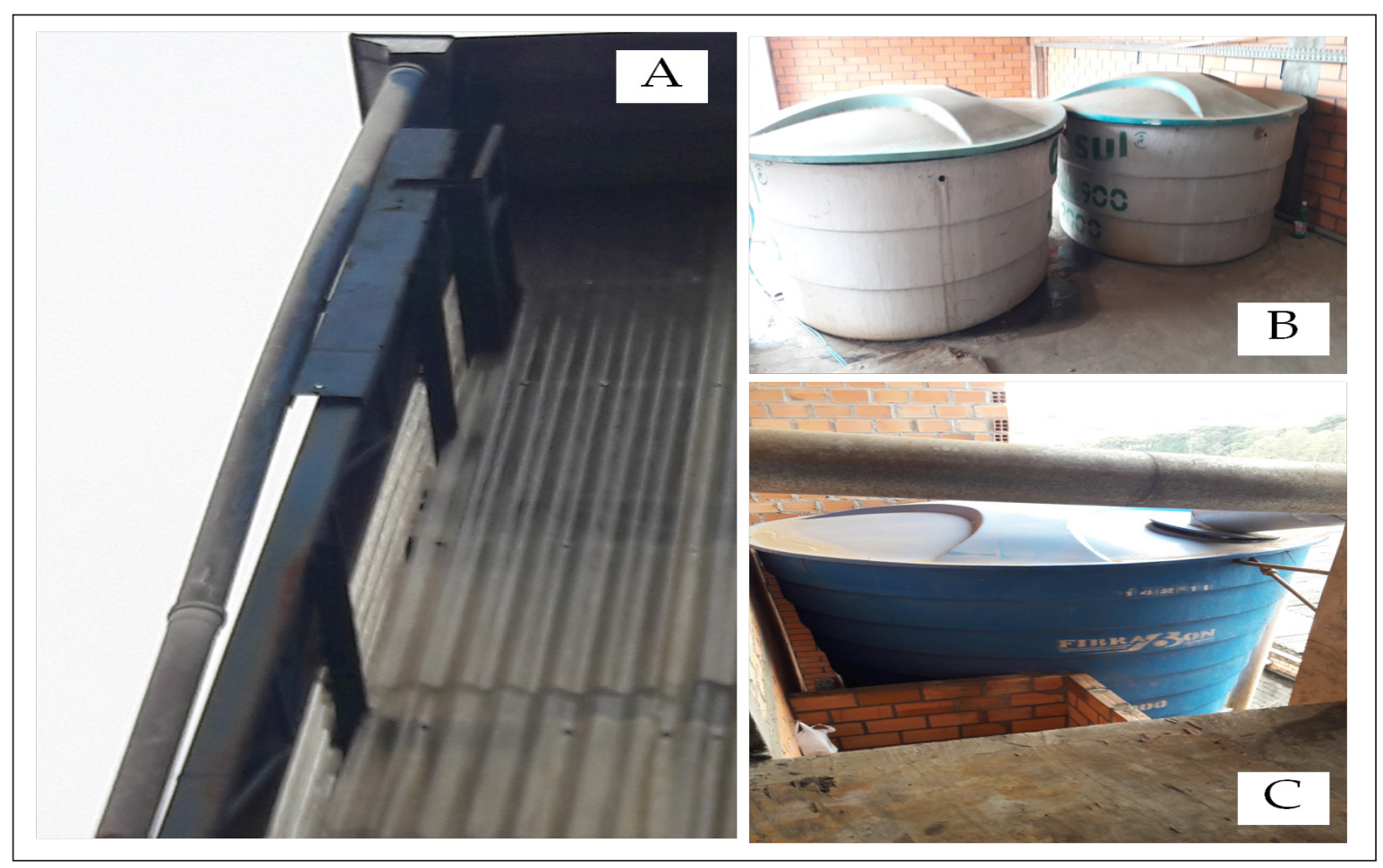

Figura 1 - Sistema de aprovechamiento del agua de lluvia: (A) Sistema de recolección del agua de lluvia; (B) Sistema de almacenamiento de agua que pasó por el sistema de enfriamiento y agua de lluvia y (C) Caja de distribución del agua reaprovechada. 


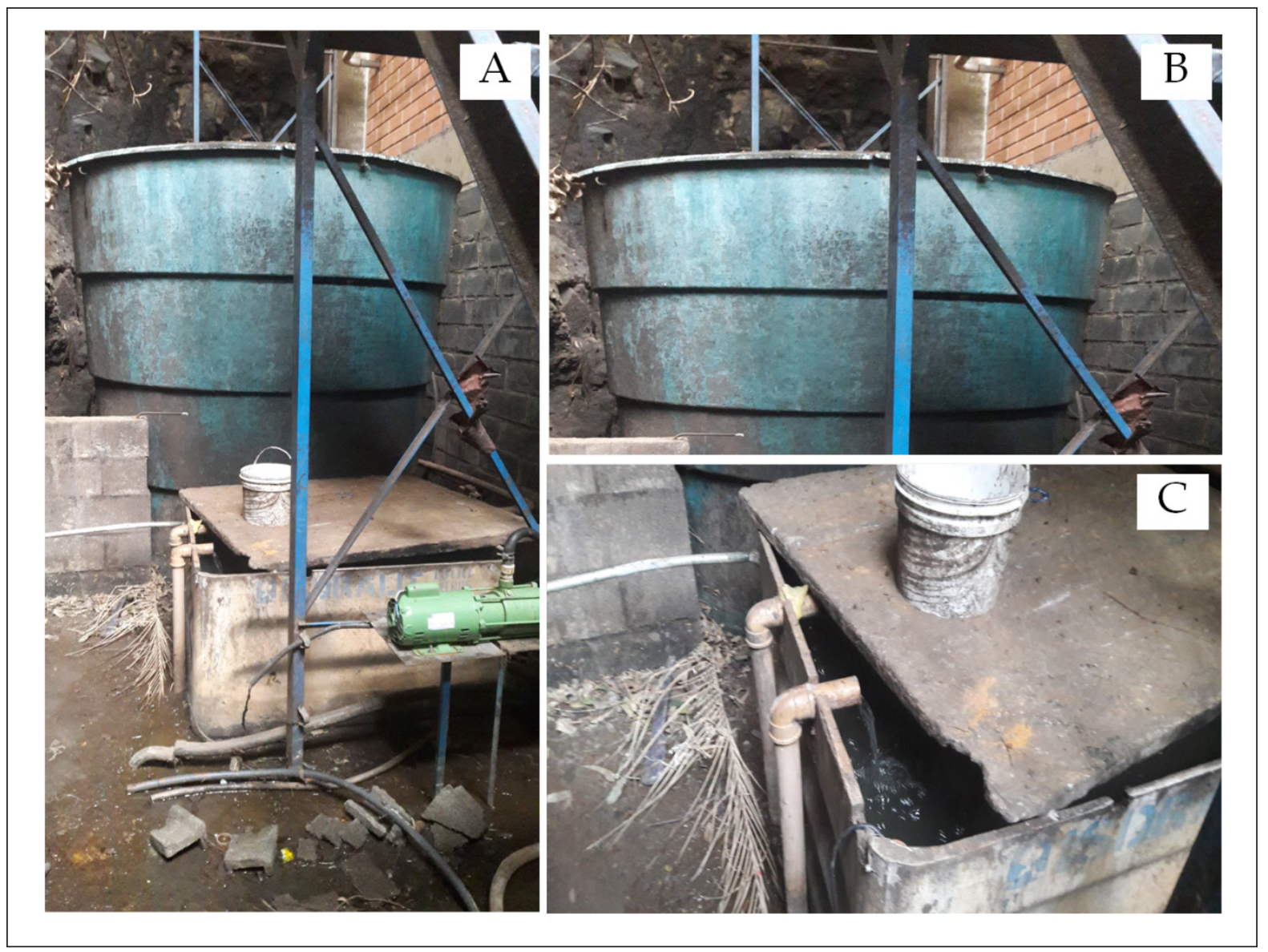

Figura 2 - Sistema de enfriamiento del efluente de la cámara instrusora componiendo un ciclo cerrado: (A) Sistema de enfriamiento; (B) Cisterna de almacenamiento y (C) Caja de enfriamiento con recirculación del efluente.

Para Fernandes, Neto y Mattos (2007) es viable económicamente y ambientalmente la utilización de sistemas de reaprovechamiento del agua de lluvia, ya que la escasez del agua es una cuestión que estamos evidenciando en la actualidad, este proceso también presenta otros beneficios indirectos como la minimización de crecidas e inundaciones, y el agua se almacena y se utiliza para el consumo. Según Minikowski y Maia (2009) el proceso de utilización de agua de lluvia debe ser un sistema complementario junto a la distribución de agua del municipio, donde si se utiliza ese sistema en meses con bajo índice pluviométrico hace imposible el proceso de utilización de agua de lluvia. De acuerdo con Nascimento, Fernandes y Yoshino (2016) es necesario desarrollar un balance hídrico para una mejor eficiencia del proceso, sin embargo, la utilización como un sistema complementario del abastecimiento de agua, presenta una alternativa viable. Ouriques et al., (2005) complementa que el aprovechamiento del agua proveniente del sistema pluvial es de suma importancia, representando un sistema que puede disminuir los gastos con la utilización de recursos.

\section{SOLVENTES}

Los residuos líquidos (solventes) generados en el proceso de lavado de piezas, baldes y bombas, son tamizados y reutilizados en el proceso industrial de la empresa y no se muestrean debido a sus características en el proceso. Esta reutilización se realiza de acuerdo con la clasificación de color del galón de tinta y se almacena en la siguiente pintura (Figura 3-B), este material se utiliza en el proceso y no necesita un destino para empresas acreditadas y licenciadas. Los disolventes y las tintas utilizadas poseen una cámara de contención para evitar cualquier fuga del producto, de acuerdo con la Figura 3. Los solventes se clasifican como Clase I por la NBR 10.004 (ABNT, 2004), obteniendo un potencial de toxicidad, corrosividad e inflamabilidad BRAGA et al., 2005). Para Kozak et al., (2008) se debe tener cuidado con el tratamiento de solventes, ya que el mismo en contacto con cualquier material, clasifica el material contaminado como un residuo peligroso. 


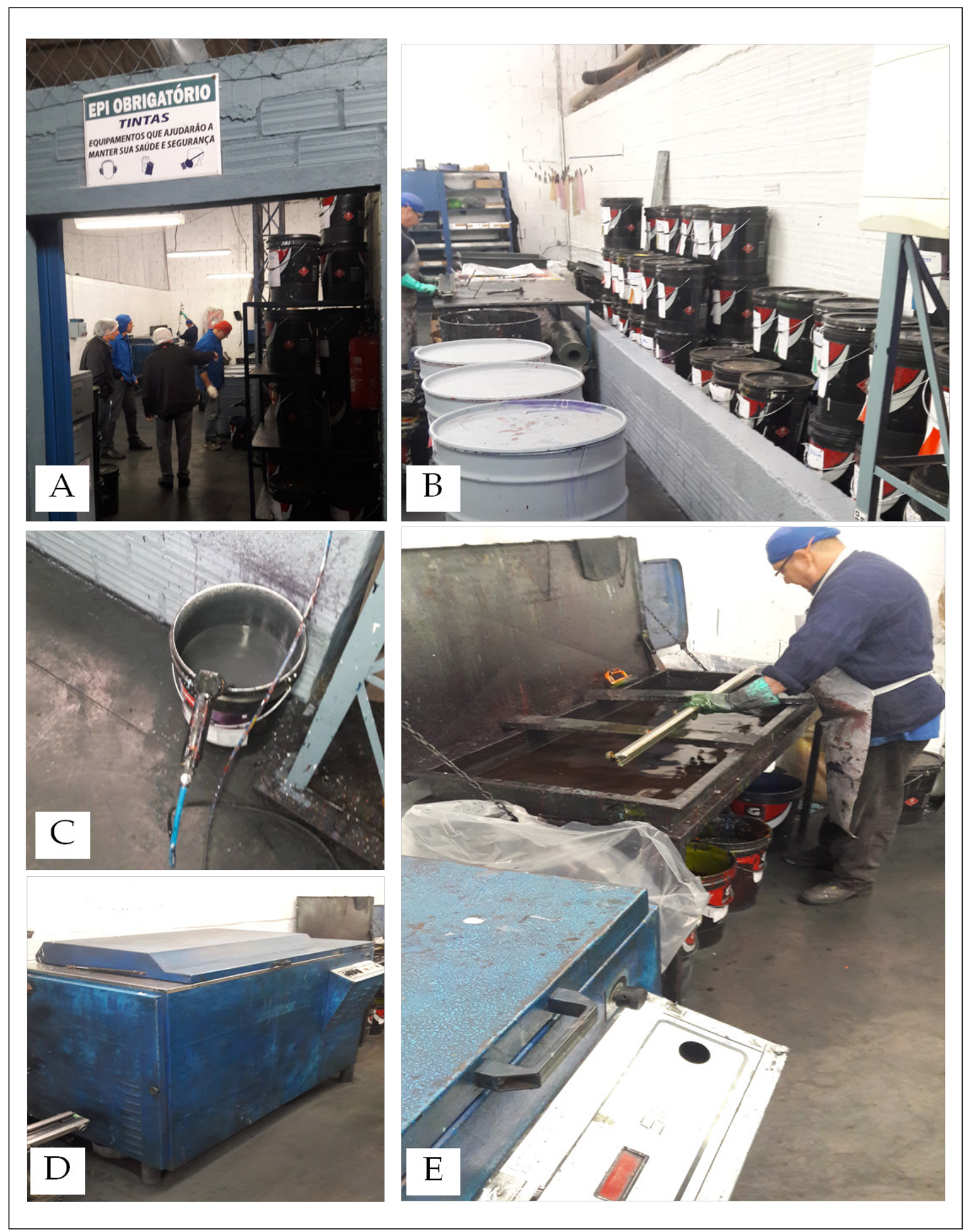

Figura 3 - Sistema de generación de residuos líquidos (disolventes): (A) lugar de lavado de piezas y manejo de disolventes; (B) almacenamiento de pinturas con adición del solvente tamizado; (C) Sistema de Lavado; (D) Bomba de lavado; (E) Lavado de piezas con resto de tinta.

Según Lobato y Lacerda (2013) es necesario monitorear y acompañar el proceso de manipulación de los solventes, ya que el mismo presenta un alto grado de toxicidad, necesitando análisis de los colaboradores que manipulan ese material. Sin embargo, según lo presentado (Figura 3.) la empresa del 
estudio posee colaboradores capacitados para desarrollar la manipulación de los solventes, como también son debidamente conscientes de la necesidad del uso del Equipo de Protección Individual - EPI's.

\section{GENERACIÓN DE RESIDUOS SÓLIDOS}

Conforme a la Figura 4. en cada sector se genera una cantidad de virutas (resto y trozos de plástico), pudiendo ser lisa o impresa. Los restos plásticos generados por la empresa proveniente del sector administrativo como el del proceso de producción son reciclados, el plástico se almacena en un lugar designado para esa operación y luego se procesa, obteniendo como producto final plástico burbuja.

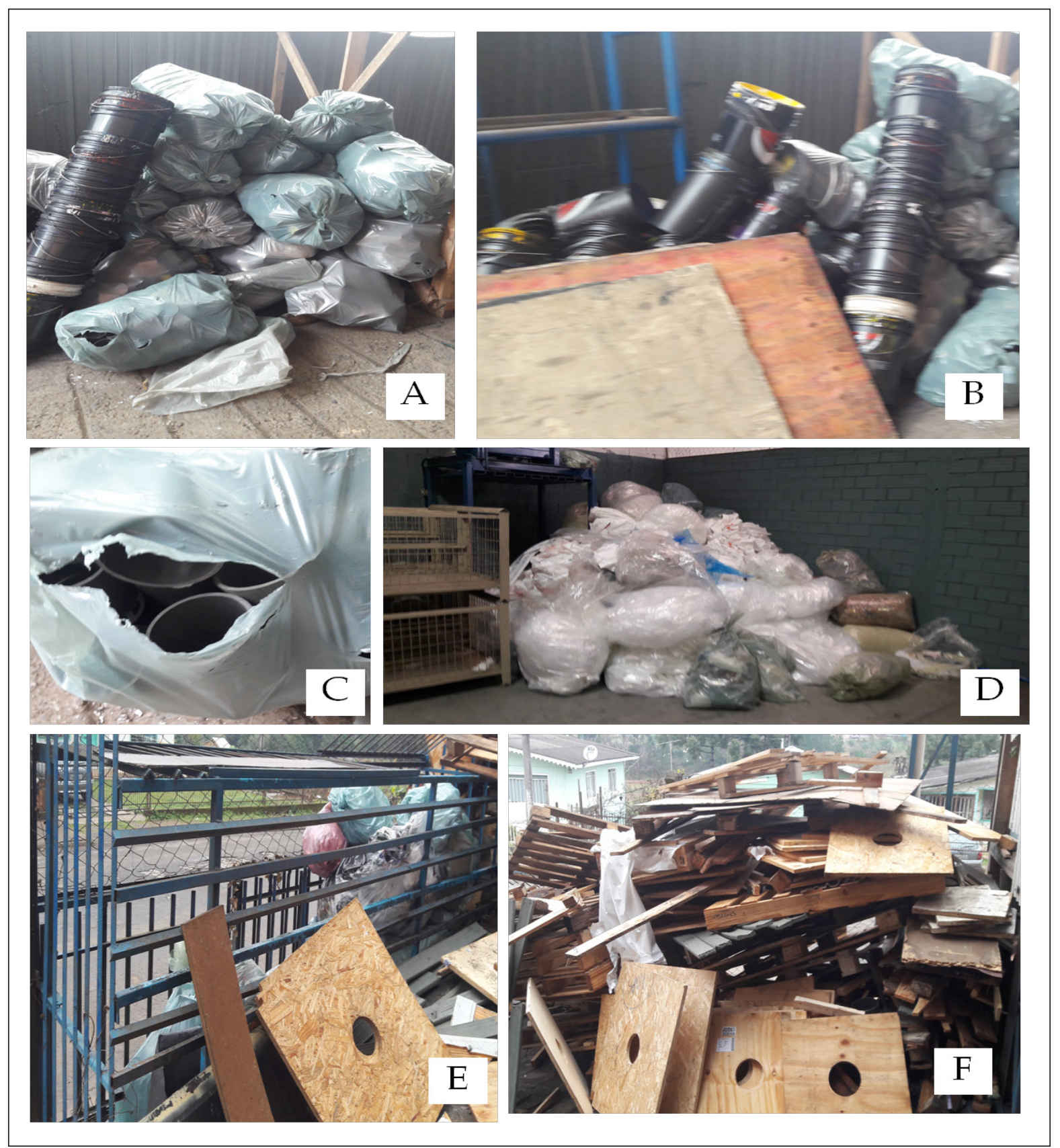

Figura 4. Principales residuos generados en el emprendimiento: (A) y (C) tubos; (B) Baldes limpios sin tinta; (D) plástico de diversos colores generados en el sector administrativo y en la producción; (E) residuos orgánicos, desechos y residuos sanitarios; (F) Madeira. 
Las estopas generadas en la empresa poseen un valor pequeño, conforme a la Tabla 2. Sin embargo, la destinación de la estopa está integrada por el sistema de logística reversa, donde una empresa licenciada realiza la recolección, lavado y tratamiento del material.

Tabla 2 - Generación de estopa en el período 2016

\begin{tabular}{c|c}
\hline \multicolumn{2}{c}{ GENERACIÓN DE RESIDUOS SÓLIDOS - ESTOPA } \\
\hline FECHA & CANTIDAD (Unidad)* \\
\hline $30 / 04 / 2016$ & $1.950,00$ \\
\hline $13 / 04 / 2016$ & 924,00 \\
\hline $07 / 06 / 2016$ & $1.505,00$ \\
\hline $10 / 06 / 2016$ & $1.288,00$ \\
\hline $24 / 06 / 2016$ & $1.170,00$ \\
\hline $03 / 08 / 2016$ & 860,00 \\
\hline $03 / 08 / 2016$ & 650,00 \\
\hline $03 / 08 / 2016$ & $1.800,00$ \\
\hline $06 / 10 / 2016$ & $1.279,00$ \\
\hline $06 / 10 / 2016$ & $1.411,00$ \\
\hline $06 / 10 / 2016$ & $1.496,00$ \\
\hline $11 / 10 / 2016$ & 833,00 \\
\hline $11 / 10 / 2016$ & $1.774,50$ \\
\hline $10 / 11 / 2016$ & $1.085,00$ \\
\hline $08 / 12 / 2016$ & 968,00 \\
\hline MEDIA MESES & $1.266,23$ \\
\hline TOTAL AÑO & 389,39 \\
\hline DESVIACION ESTANDAR & \\
\hline *alores obtenidos por el certificado emitido por la empresa receptora y destinataria del residuo.
\end{tabular}

El papel y cartón se destinan al sistema de recolección selectiva del municipio, esta recolección es realizada por COOCIMA, pero debido a las características del lugar donde se almacena, muchos residentes de la zona circundante y recolectores de materiales reciclables acaban recogiendo ese material. Campos y Borga (2015a), Campos y Borga (2016) describen que todo material potencialmente reciclable es recogido por el programa de recolección selectiva en área urbana y rural del municipio y está destinado a una cooperativa que hacen la clasificación y reciclan ese material. Según Lomasso et al., (2015), Campos y Borga (2015b) el proceso de reciclaje a través de la recolección selectiva disminuye el impacto ambiental y favorece el desarrollo sostenible de un municipio.

Además del plástico, estopa, papel y cartón la empresa posee la generación de resto de tubos, madera (paletas), baldes de tinta, adhesivo de embalaje, residuos orgánicos y sanitarios. Los residuos orgánicos y residuos sanitarios son destinados al sistema de recolección convencional del municipio de Caçador. Según Almeida (2012) todo material orgánico y residuos sanitarios que se recogen en el área urbana y rural del municipio se destinan a un relleno sanitario.

El resto de tubos, madera y baldes de tinta son encaminados al sector de reciclaje de la empresa denominada Recrear, que tritura y destina correctamente el material. La madera (paletas) generada en el proceso es destinada al sector de reciclaje de la empresa donde las paletas con defectos son reestructuradas e insertadas en la empresa nuevamente. Para Mano, Pacheco y Bonelli (2010) el proceso de reciclado es un mecanismo que busca reducir el destino incorrecto de los residuos, ya que el mismo se transforma en un nuevo producto, minimizando la cantidad de recursos naturales y, consecuentemente, disminuye los impactos ambientales ocasionados por la acción antropogénica.

Los adhesivos de embudo y EPI's se almacenan en un lugar apropiado y se encaminan a la empresa licenciada para recoger y disponer sobre los residuos, siendo esta una colecta realizada anualmente, la cual, durante el estudio, obtuvo la generación de 2.202,00 kg.año-1.

El estudio presenta que la empresa presenta conformidad ambiental en su proceso productivo. Para Selbach y Naime (2014) esta cuestión se asigna a las empresas para buscar estar de acuerdo con las exigencias del mercado. Según Huerta y García (2009) el proceso de conducir las estrategias 
ambientales es un método interactivo, creativo y participativo que encamina claramente a acciones y compromisos de una empresa en el futuro, ya que esta interacción proporciona el desarrollo de planes, programas y proyectos a corto y a largo plazo.

\section{CONCLUSIONES}

A partir del estudio se puede alcanzar el objetivo del trabajo de caracterizar los controles ambientales adoptados por la empresa a estudio mediante su proceso productivo y etapas de generación y adquisición de materiales.

El proceso de monitoreo de los condicionantes ambientales empleados en las licencias ambientales es un proceso que ayuda en la minimización de los impactos ambientales generados por las empresas, ya que es un proceso de fiscalización ambiental, en el cual la empresa debe cumplir con las exigencias legales para poder recibir la siguiente licenciay una licencia ambiental y válida para cuatro años.

El sistema de utilización de agua de lluvia es un proceso que ayuda en las cuestiones financiera y ambiental de una empresa, donde la empresa acaba utilizando menos agua proveniente del sistema de distribución pública y, consecuentemente, disminuye el valor de la factura de agua.Ese proceso ayuda al sistema de drenaje pluvial del municipio, pues el agua queda retenida en la empresa y no en las calles, pudiendo evitar crecidas e inundaciones.

La empresa a estudio genera impactos ambientales significativos en el medio ambiente, pero la misma busca minimizar esta cuestión, donde, además del pensamiento ambiental de los emprendedores de la empresa del estudio necesita cumplir las exigencias de sus consumidores, dado que muchos clientes de la empresa buscan una empresa licenciada y con un proceso productivo que genere menos atenuantes ambientales.

Los residuos sólidos son el principal aspecto negativo ambiental generado por la empresa, aunque la empresa del presente trabajo dispone de una gestión eficiente en esta cuestión, ya que todos sus residuos se destinan correctamente o tienen el proceso de reciclaje en la propia empresa. Sin embargo, para ayudar en las cuestiones ambientales la empresa necesita entrenar y capacitar a sus empleados para buscar una menor generación de residuos, y, principalmente, mejorar la gestión ambiental de la empresa.

Aunque no se analizado, es necesario desarrollar el monitoreo del sistema de alcantarillado sanitario, a través del análisis de la eficiencia de la fosa y filtro proveniente de la empresa, puesestá recogiendo un recurso natural para el consumo y destinando un efluente con una carga alta de material orgánico, que se dirige al sistema de drenaje del municipio y posteriormente al Río do Peixe. También, como en los efluentes, es necesario desarrollar el monitoreo del ruido, ya que la empresa posee máquinas y la misma genera una presión sonora, pese a que la empresa posee un técnico de seguridad que controla la gestión de la entrega y recolección del EPI's.

Así, se puede concluir que la empresa del estudio está en conformidad con las cuestiones ambientales (tratamiento de aguas residuales sanitarias y gestión de los residuos sólidos y líquidos); sin embargo, es necesario desarrollar el monitoreo y gestión continua de los mismos, no buscando solamente cumplir con lo establecido por la licencia ambiental de operación y sí minimizar los impactos ambientales.

\section{REFERENCIAS}

ALMEIDA, R. G.. Estudo de geração de resíduos sólidos domiciliares urbanos no município de Caçador-SC, á partir da caracterização física e composição gravimétrica. Ignis: Periódico Científico de Arquitetura e Urbanismo, Engenharias e Tecnologia da Informação, Caçador, v.1, n.1, p. 71-92, 2012.

ANJOS, W.F.; NÓBREGA, W.R.; SILVEIRA, J.A.R.; SILVA, M.D. Urbanização dispersa e a transformação de paisagem natural: Estudo de caso sobre a dispersão urbana na bacia do Rio Cuiá, na cidade de João Pessoa - PB, Brasil. GEOAMBIENTE ON-LINE, Jataí, v.28, n.1, p.127-141, Jan./Jun. 2017. 
ASSOCIAÇÃO BRASILEIRA DE NORMAS TÉCNICAS. NBR 7229: Projeto, construção e operação de sistemas de tanques sépticos. Rio de Janeiro, 1993.

BERNARDO, J.S.S.; CAMAROTTO, J.A. Fatores motivadores da adoção de práticas ambientais em empresas paulistas processadoras de madeira. Produção, São Carlos, v.22, n.1, p.173-184, Jan./Fev. 2012.

BOGARIM, E.P.A.; PAULUS, L.A.R.; CABREIRA, R.P.S.; LENIS, J.N.R.; DIAS, A.P.A. Qualidade ambiental em conformidade com o processo de licenciamento sob a visão de um gestor ambiental - Ênfase nos estabelecimentos de lavagem automotiva. Revista Eletrônica em Gestão, Educação e Tecnologia Ambiental REGET, Santa Maria, v.18, n.1, p.1-6, Abri. 2014.

BORELLA, I.E.; NAIME, R. Transformar a gestão ambiental integrada em vantagem competitiva. Revista Eletrônica em Gestão, Educação e Tecnologia Ambiental - REGET, Santa Maria, v.6, n.6, p.1025-1042, 2012.

BRAGA, B.; HESPANHOL, I.; CONEJO, J.G.L.; MIERZWA, J.C.; BARROS, M.T.L.; SPENCER, M.; PORTO, M.; NUCCI, N.; JULIANO, N.; EIGER, S. Introdução à engenharia ambiental: O desafio do desenvolvimento sustentável. 2. ed. São Paulo: Pearson Prentice Hall, 2005.

CAMPOS, R.F.F.; COSTA, D.D. Análise do impacto ambiental pela dispersão de poluentes atmosféricos, através da queima de resíduos. InterfacEHS - Saúde, Meio Ambiente e Sustentabilidade, São Paulo, v.12, n.1, p.182-191, Jan./ Jun. 2017.

CAMPOS, R.F.F.; BORGA, T. Levantamento da geração anual dos resíduos sólidos recicláveis do município de Caçador-SC. Revista Monografias Ambientais - REMOA, Santa Maria, v.15, n.1, p.209-2019, Jan./Abr. 2016.

CAMPOS, R.F.F.; BORGA, T. Caracterização gravimétrica do material Reciclável Destinado á COOCIMA pelo programa de coleta seletiva do município de Caçador-SC. Revista Eletrônica em Gestão, Educação e Tecnologia Ambiental - REGET, Santa Maria, v.19, n.3, p.325-338, Set./Dez. 2015a.

CAMPOS, R.F.F.; BORGA, T. Geração de resíduos recicláveis no município de Caçador-SC. Ignis: Periódico Científico de Arquitetura e Urbanismo, Engenharias e Tecnologia da Informação, Caçador, v.4, n.1, p.24-38, Jan./Dez. 2015b.

CAMPOS, R.F.F.; LIMA, C. Sustentabilidade através de remanejo de resíduos com pratica de gestão ambiental implantado no Supermercado Cereal. Ignis: Periódico Científico de Arquitetura e Urbanismo, Engenharias e Tecnologia da Informação, Caçador, v.3, n.1, p.24-44, Jan./Dez. 2014.

COSTA, S.L.; SOARES. M.J.N.; PESSOAS, F.M.G. Sistema jurídico brasileiro de licenciamento ambiental. Revista Ibero-Americana de Ciências Ambientais, Aquidabã, v.6, n.2, p.7-22, Jun./Nov. 2015.

D’AGOSTIN, A.; GLUSCZAK, A.G.; LAVNITCKI, L.; BECEGATO, V.A. Caracterização de áreas degradadas por atividade antrópica. GEOAMBIENTE ON-LINE, Jataí, v.28, n.1, p.80-92, Jan./Jun. 2017.

FERNANDES, D.R.M.; NETO, V.B.M.; MATTOS, K.M.C. Viabilidade econômica do uso da água da chuva: Um estudo de caso da implantação de cisterna na UFRN/RN. In: XXVII Encontro nacional de Engenharia da Produção, Foz do Iguaçu, p.1-9, 2007.

GIRARDI, G.; SELLITTO, M.F. Medição e reconhecimento do risco físico ruído em uma empresa da indústria moveleira da serra gaúcha. Estudos Tecnológicos, v.7, n. 1, p.12-23, jan/abr, 2011.

HALBERSTADT, K.F.; SILVA, V.A.; SANTOS, M.B.; SCHERE, F.L.; CARPES, A.M.; OLIVEIRA, M.C.S.F. Práticas sustentáveis na destinação dos resíduos resultantes da cadeia produtiva de arroz. Revista Eletrônica em Gestão, Educação e Tecnologia Ambiental - REGET, Santa Maria, v.19, n.3, p.298-312, Set./ Dez. 2015. 
HERTA, E.; GARCÍA, J. Estrategias de gestión ambiental: Una perspectiva de las organizaciones modernas. Clío América, Enero, v.3, n.3, p.15-30, Jun. 2009.

KOZAKA, P.A.; CORTEZB, A.M.; SCHIRMERC, W.N.; CALDEIRAD, M.V.W.; BALBINOTE, R. Identificação, quantificação e classificação dos resíduos sólidos de uma fabrica de moveis. Rev. Acad., Ciênc. Agrár. Ambient.,Curitiba, v.6, n.2, p. 203-212, Abr./Jun. 2008.

LAZAROTTO, D.; IBDAIWI, T.K.R.; ALMEIDA, D.M.; COSTA, V.M.F.; LOPES, L.F.D.; SANTOS, R.C.T. Gestão Ambiental sob a Ótica de um Centro Automotivo: um estudo de caso na cidade de Santa Maria/RS. Revista Eletrônica em Gestão, Educação e Tecnologia Ambiental - REGET, Santa Maria, v.18, n.2, p.846-858, Mai./Ago. 2014.

LEANDRO, L.A.; NEFFA, E.M. Soluções tecnológicas ecoeficientes para o desenvolvimento sustentável: uma análise teórico-conceitual. Revista Eletrônica em Gestão, Educação e Tecnologia Ambiental - REGET, Santa Maria, v.18, n.1, p.566-576, Abri. 2014.

LOBATO, D.C.B.; LACERDA, A.B.M. Efeitos auditivos dos solventes:revisão de literatura.Tuiuti: Ciência e Cultura, Ciritiba, n.47, p. 67-50, 2013.

LOMASSO, A.L.; DOS SANTOS, B.R.; ANJOS, F.A.S.; ANDRADE, J.C.; SILVA, J.C.; DOS SANTOS, Q.R.; CARVALHO, A.C.M. Benefícios e desafios na implementação da reciclagem: em estudo de caso no Centro Mineiro de Referência em Resíduos (CMRR). Revista Pensar Gestão e Administração, v. 3, n. 2, p.120, Jan. 2015.

MANO, E.B.; PACHECO, E.B.A.V.; BONELLI, C.M.C. Meio ambiente, poluição e reciclagem. $2^{\mathrm{a}}$ ed. - São Paulo:Bucher, 2010.

MINIKOWSKI, M.; MAIA, A.G. Sistema de aproveitamento de água da chuva no município de Irati PR.Rev. Acad., Ciênc. Agrár. Ambient., Curitiba, v.7, n.2, p.181-188, Abr./Jun. 2009.

MONARO, D.L.G.; PLENS, A.C.O.; MONARO, R.L.G.; ASSUMPÇÃO, M.R.P. A Responsabilidade socioambiental como uma estratégia empresarial em empresas de pequeno porte do setor metal mecânico. Revista Eletrônica em Gestão, Educação e Tecnologia Ambiental - REGET, Santa Maria, v.19, n.1, p.129-138, Jan./ Abr. 2015.

NASCIMENTO, T.V.; FERNANDES, L.L.; YOSHINO, G.H. Potencial de aproveitamento de água de chuva na Universidade Federal do Pará - Belém/PA. Revista Monografias Ambientais - REMOA, Santa Maria, v.15, n.1, p.105-116, Jan./Abr. 2016.

OURIQUES, R.Z.; SCHNEIDER, A.; LOPES, M.I.P.; BARROSO, L.B. Aproveitamento da água de chuva em escola municipal de Santa Maria-RS.Disc. Scientia. Série: Ciências Naturais e Tecnológicas, Santa Maria, v. 6, n. 1, p.1-10, 2005.

PAZ, Y.M.; MORAIS, M.M.; HOLANDA, R.M. Desenvolvimento Econômico Regional e o Aproveitamento de Resíduos Sólidosno Polo da Indústria da Cerâmica Vermelha do Estado de Pernambuco. Revista Brasileira de Geografia Física, v.6, n.6, p.1682-1704, 2013.

PEREIRA, R.S. Poluição Hídrica: Causa e consequências. Revista Eletrônica de Recursos Hídricos, IPH UFRGS, v.1, n.1, p.20-36, 2004.

PIMENTA, H.C.D.; TORRES, F.R.M.; RODRIGUES, B.S.; ROCHA-JÚNIOR, J.M. O esgoto: A importância do tratamento e as opções tecnológicas. In: XXII Encontro Nacional de Engenharia de Produção, Curitiba, p.1-8, 2002. 
SANTOS, A.R.L.; AQUINO, S.F.; CARVALHO, C.F.; VIEIRA, L.A.; GONTIJO, E.S.J. Caracterização e tratabilidade biológica dos efluentes líquidos gerados em cabines de pintura de uma indústria moveleira. Eng Sanit Ambient, v.15, n.4, p.357-366, Out./Dez. 2010.

SELBACH, E.; NAIME, R. Diagnóstico de sustentabilidade em indústria moveleira do RS: Estudo de caso em fábrica no Vale do Caí. Revista Eletrônica em Gestão, Educação e Tecnologia Ambiental - REGET, Santa Maria, v.18, n.2, p.312-328, Abri. 2014.

SILVA, N.A.; FURTADO, O.S.; DIAS, W.S.; SELLITTO, M.A. Avaliação de desempenho ambiental em uma empresa da indústria cerâmica de Tocantins. Revista Eletrônica em Gestão, Educação e Tecnologia Ambiental - REGET, Santa Maria, v.19, n.21, p.848-61, Mai./Ago. 2015.

SILVA, L.O.; PEREIRA, A.A. Caracterização do sistema de tratamento de esgoto biológico como o uso de membrana filtrante em uma empresa do setor automobilístico. Revista de Magistro de Filosofia, Anápolis, v.7, n.14, p.22-36, Jul./ Dez. 2014.

SISINNO, C.L.S. Disposição em aterros controlados de resíduos sólidos industriais não-inertes: avaliação dos componentes tóxicos e implicações para o ambiente e para a saúde humana. Caderno de Saúde Publica, Rio de Janeiro, v.19, n.2, p.369-374, Mar./Apr. 2003.

WHITMAN, B.E.; MIHELCIC, J.R.; MAYER, A.S. Fornecimento e distribuição de água e coleta de esgoto. In: MIHELCIC, J.R.; ZIMMERMAN, J.B. Engenharia Ambiental: Fundamentos, Sustentabilidade e Projetos. Rio de Janeiro: LTC. 2015. 